\title{
Radiometric studies on the use of selective inhibitors in the identification of Mycobacterium spp.
}

\author{
T. COLLINS and P. N. LEVETT*
} Department of Medical Microbiology, Regional Hospital, Galway, Eire and *Department of Biological and
Biomedical Sciences, University of Ulster, Coleraine, Co. Derry BT52 1SA, Northern Ireland

\begin{abstract}
Summary. Radiometric selective inhibition tests were developed and evaluated for the rapid differentiation of Mycobacterium spp. Both a $\rho$-nitrobenzoic acid (PNB) test and a commercially-prepared $\rho$-nitro- $\alpha$-acetylamino- $\beta$-hydroxypropiophenone (NAP) test successfully differentiated $M$. tuberculosis and $M$. bovis from "atypical" mycobacteria or mycobacteria other than tubercle bacilli (MOTT). Thiophene-2carboxylic acid hydrazide ( $\mathrm{TCH}$ ) readily distinguished human $M$. tuberculosis strains from $M$. bovis, irrespective of resistance to isoniazid. Both PNB and TCH tests were utilised in a routine radiometric susceptibility testing scheme over a period of 1 year in which 110 isolates of $M$. tuberculosis, 10 of $M$. bovis and one isolate of BCG were correctly differentiated from 10 isolates of MOTT. The rapidity, sensitivity and specificity of these radiometric tests can play a useful role in mycobacterial identification.
\end{abstract}

\section{Introduction}

The so called "atypical" mycobacteria, or mycobacteria other than tubercle bacilli (MOTT), are well established pathogens of man. They normally require some pre-existing condition such as bronchitis or emphysema before they can cause pulmonary disease and the immunocompromised patient is particularly prone to infection. Predictably, patients with the acquired immune deficiency syndrome are a group in whom infections with MOTT are appearing with increasing frequency (Jenkins, 1987; Lévy-Frébault et al., 1987; Males et al., 1987).

The growth of some of the MOTT on conventional media is slow and it can be many weeks between receipt of the specimen and production of the final report. Whilst this does not necessarily affect the clinical management of the patient, clearly a more rapid method of isolation and identification is desirable. The Bactec $460^{\circledR}$ (Johnston Laboratories) detects the growth of mycobacteria by measuring the evolution of ${ }^{14} \mathrm{C}$-labelled $\mathrm{CO}_{2}$ from a ${ }^{14} \mathrm{C}$-labelled substrate, the degree of growth being expressed as a growth index (GI). With this system, positive results are obtained in

Received 26 April 1988; accepted 1 Sep. 1988.

* Present address: University of the West Indies, Faculty of Medical Sciences, Queen Elizabeth Hospital, St Michael, Barbados. between 7 and 12 days but the positive GI only indicates the presence of a mycobacterium and further tests are necessary to differentiate between the tuberculosis complex and MOTT.

Conventional mycobacterial identification has for many years been based upon the use of selective inhibitors for the differentiation of Mycobacterium tuberculosis and $M$. bovis from the MOTT (Marks, 1976). Resistance of $M$. tuberculosis and M. bovis to $\rho$-nitrobenzoic acid (PNB) and hydroxylamine is well established (Tsukamura and Tsukamura, 1964; Tsukamura, 1965, 1970). The resistance of some strains of $M$. kansasii to PNB has also been noted (Tsukamura, 1984). Hydroxylamine would appear to offer no advantage over PNB as a screening agent (Collins and Yates, 1979).

The differential properties of $\rho$-nitro- $\alpha$-acetylamino- $\beta$-hydroxypropiophenone (NAP), described by Eidus et al. (1960), have rarely been used in conventional screening techniques. However NAP inhibition has been adapted to radiometric techniques and has been found to be rapid and sensitive (Laszlo and Siddiqi, 1984).

Thiophene-2-carboxylic acid hydrazide (TCH) is useful in differentiating the classical, African and Asian variants of $M$. tuberculosis (Harrington and Karlson, 1966). The classical human variant is resistant to $\mathrm{TCH}$ irrespective of resistance to isoniazid, whereas all other variants are susceptible, as is $M$. bovis (Yates et al., 1984). 
The purpose of this investigation was to develop radiometric selective inhibition tests and investigate their value in the routine screening and susceptibility testing of clinical isolates of mycobacterial species.

\section{Materials and methods}

\section{Mycobacterial strains}

The following were obtained from the National Collection of Type Cultures, Colindale Avenue, London: $M$. avium NCTC 10437, $M$. bovis NCTC 10772, $M$. flavescens NCTC 10271, $M$. fortuitum NCTC 10394, $M$. gordonae NCTC 10267, $M$. kansasii NCTC 10268, $M$. scrofulaceum NCTC 10803 and $M$. tuberculosis NCTC $7416(\mathrm{H} 37 \mathrm{Rv})$. Three isoniazid-resistant strains of $\boldsymbol{M}$. tuberculosis, labelled TB1, TB2 and TB3, and three strains of $M$. kansasii (B1, B2 and B3) were received from $\mathbf{M}$. Chadwick, Brompton Hospital, London. Further isolates of $M$. kansasii were received from P. A. Jenkins, PHLS Mycobacterium Reference Unit, Cardiff (strain C1) and from C. Byrne, Peamount Hospital, Dublin (strain P1). Three strains of $M$. bovis from bovine para-tracheal lymph nodes and three strains of $M$. tuberculosis from human sputum, isolated in this laboratory, were also studied.

Over a period of 1 year 131 fresh clinical isolates were studied, comprising strains of $M$. tuberculosis (110 isolates), $M$. bovis (10), BCG (1), M. kansasii (2), $M$. fortuitum (2), M. avium-intracellulare (3), $M$. gordonae (2) and $M$. xenopi (1). All isolates were identified by conventional methods (Marks, 1976).

During this study strains were maintained at $-70^{\circ} \mathrm{C}$ in 2-rnl volumes of Middlebrook 7H9 Medium (Difco).

\section{Media}

Media for conventional identification methods were prepared as described by Marks (1976). LowensteinJensen (LJ) slopes containing TCH (Biomerieux) $5 \mu \mathrm{g} /$ $\mathrm{ml}$ or PNB (Mast Laboratories) $500 \mu \mathrm{g} / \mathrm{ml}$ were stored at $4^{\circ} \mathrm{C}$ before use. Bottles containing $4 \mathrm{ml}$ of Bactec 12B Medium, labelled with ${ }^{14} \mathrm{C}$-palmitic acid, were obtained from Johnston Laboratories Inc., Towson, MD, USA.

\section{Reagents}

$\mathrm{TCH}$ (Sigma) was dissolved in ethanol $50 \% \mathrm{v} / \mathrm{v}$ to a concentration of $200 \mu \mathrm{g} / \mathrm{ml}$ and was then diluted with sterile distilled water to a final concentration of $50 \mu \mathrm{g} / \mathrm{ml}$. Immediately before use, $0 \cdot 1 \mathrm{ml}$ of this solution was added aseptically to $4 \mathrm{ml}$ of Bactec 12B medium, giving a final concentration of approximately $1 \cdot 2 \mu \mathrm{g} / \mathrm{ml}$.

To $1 \mathrm{~g}$ of PNB (Sigma) dissolved in $10 \mathrm{ml}$ of $1 \mathrm{~N}$ aqueous $\mathrm{NaOH}$ was added $70 \mathrm{ml}$ of sterile distilled water and a few drops of phenol red $0 \cdot 1 \% \mathrm{w} / \mathrm{v}$. This solution was then neutralised by the addition of $1 \mathrm{~N}$ aqueous $\mathrm{HCl}$, made up to $100 \mathrm{ml}$ with sterile distilled water and autoclaved at $121^{\circ} \mathrm{C}$ for $10 \mathrm{~min}$. The sterile solution was stored at $4^{\circ} \mathrm{C}$ before use. From this PNB stock solution $0.2 \mathrm{ml}$ was added to 4-ml volumes of Bactec 12B medium immediately before inoculation, giving a final PNB concentration of $c .500 \mu \mathrm{g} / \mathrm{ml}$.

Glass vials, each containing a paper disk impregnated with NAP $5 \mu \mathrm{g}$, were obtained commercially (Johnston Laboratories).

\section{Radiometric tests}

All cultures for radiometric testing were incubated at $37^{\circ} \mathrm{C}$ in an aerobic atmosphere containing $\mathrm{CO}_{2} 10 \%$. The amount of growth was determined daily for 5 days with a Bactec 460TB instrument (Johnston Laboratories). The amount of ${ }^{14} \mathrm{CO}_{2}$ liberated was directly related to the metabolism of the labelled substrate and to the growth of the mycobacterial culture. The degree of growth was expressed in terms of the growth index (GI).

Cultures of mycobacteria to be treated in both conventional and radiometric selective inhibition tests were grown in $4 \mathrm{ml}$ of Bactec 12B medium until a growth index of 999 or greater was achieved; $0 \cdot 1-\mathrm{ml}$ volumes were then used to inoculate conventional $\mathrm{LJ}$ slopes and test vials containing the inhibitory agents and control vials without inhibitors. The NAP inhibition test was inoculated as described elsewhere (Morgan et al., 1985).

The following criteria were used to interpret growth patterns in selective inhibition tests:

(a) two consecutive daily decreases in GI after $48 \mathrm{~h}$ and a continuing GI decrease = susceptible;

(b) slight GI increase in the first $48 \mathrm{~h}$ and then no increase $=$ susceptible;

(c) increased daily GI reading to $>400=$ resistant;

(d) no increase or slight decrease in GI in the first $48 \mathrm{~h}$ and then three consecutive daily increases $=$ resistant.

\section{Results}

\section{Reference strains}

$P N B$. Reference strains representing eight mycobacterial species were examined for susceptibility to PNB. $M$. avium NCTC $10437, M$. flavescens NCTC $10271, M$. fortuitum NCTC 10394, $M$. gordonae NCTC 10267 and $M$. scrofulaceum NCTC 10803 each showed a rapid increase in GI over a 5day period (fig. 1). In contrast, $M$. bovis NCTC 10772, M. tuberculosis NCTC 7416 and $M$. kansasii NCTC 10268 were markedly inhibited by PNB.

The susceptibility of another five $M$. kansasii isolates and strain NCTC 10268 to PNB was studied further (fig. 2). NCTC 10268 was again inhibited by PNB in the radiometric test as was strain B2; both yielded only very light growth on LJ-PNB slopes. One isolate (B3) grew slowly in the radiometric medium and produced a light growth on the LJ-PNB slope. The remaining isolates 

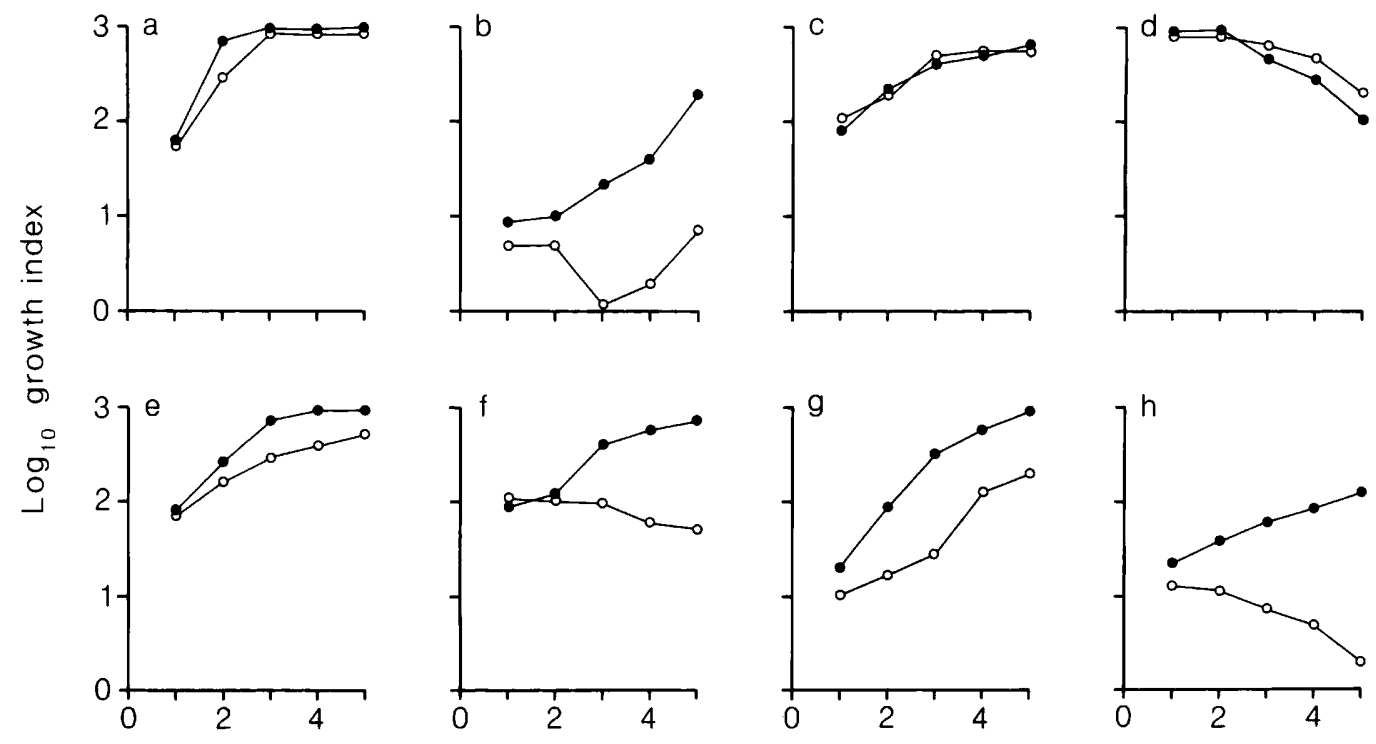

Days

Fig. 1. Susceptibility of mycobacteria to $\rho$-nitrobenzoic acid (PNB): (a) $M$. avium NCTC 10437; (b) $M$. bovis NCTC 10772; (c) $M$. flavescens NCTC 10271; (d) M. fortuitum NCTC 10394; (e) M. gordonae NCTC 10267; (f) M. kansasii NCTC 10268; (g) M. scrofulaceum NCTC 10803; (h) $M$. tuberculosis NCTC 7416; control (O) and PNB $500 \mu \mathrm{g} / \mathrm{ml}$ (O).
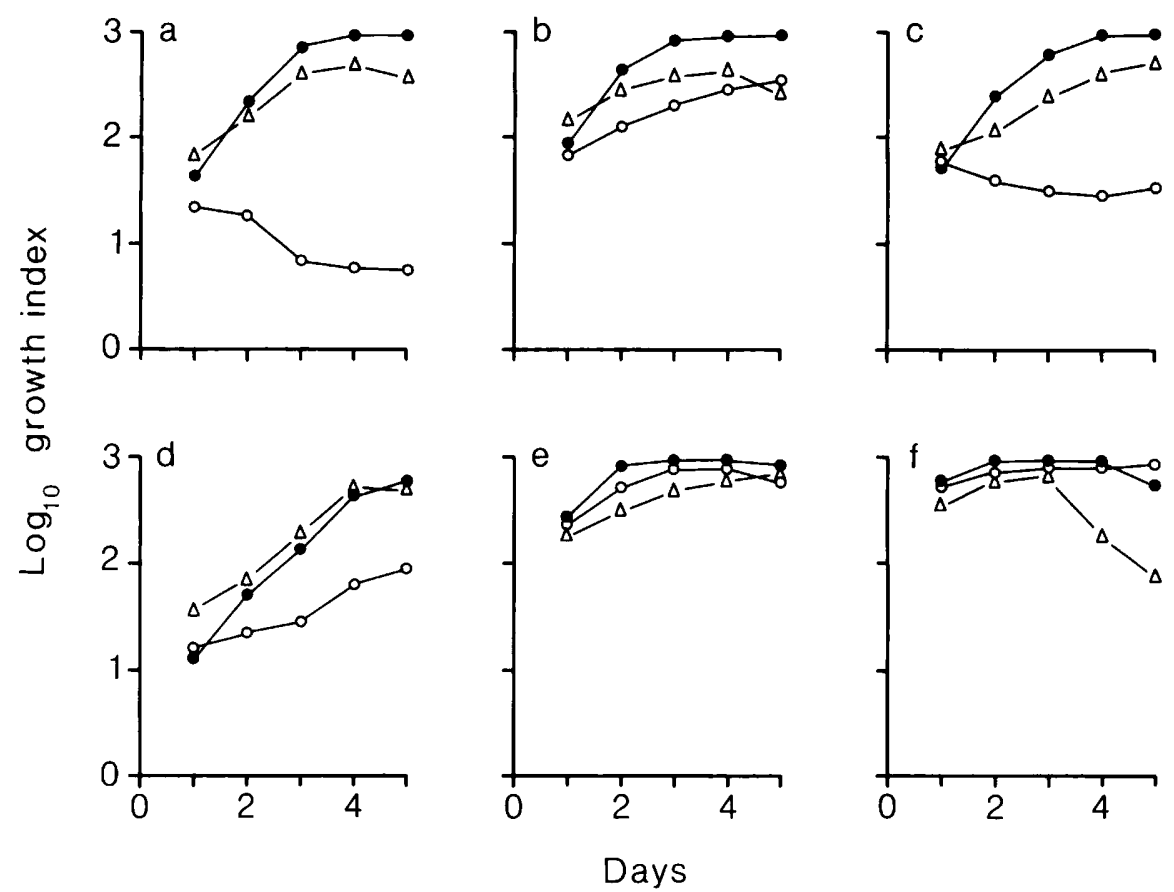

Fig. 2. Growth of $\operatorname{six} M$. kansasii strains in the presence of $\rho$-nitrobenzoic acid (PNB) $(\bigcirc)$ or $\rho$-nitro- $\alpha$-acetylamino- $\beta$ hydroxypropiophenone (NAP) $(\triangle)$ and control (O): (a) NCTC 10268; (b) B1; (c) B2; (d) B3; (e) C1; (f) P1. 

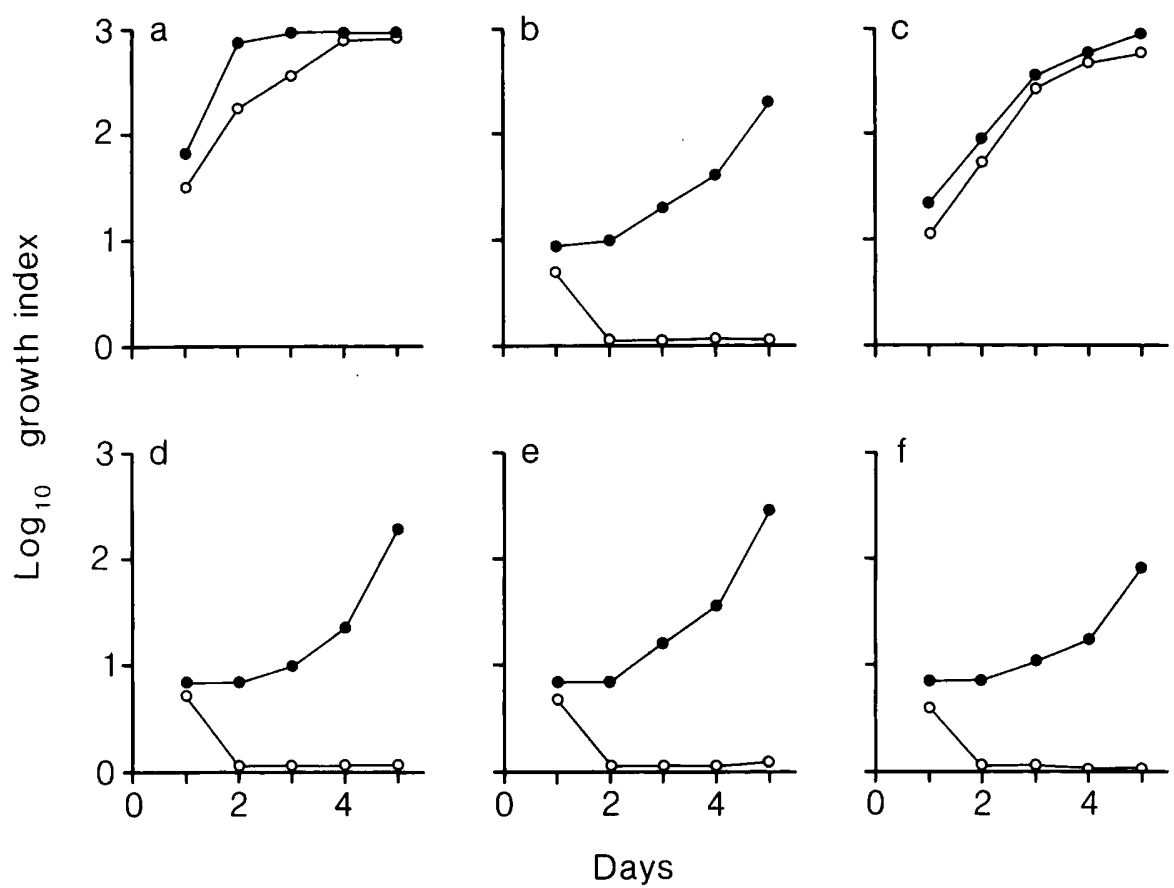

Fig. 3. Susceptibility of mycobacteria to $\rho$-nitro- $\alpha$-acetylamino- $\beta$-hydroxypropiophenone (NAP): (a) $M$. avium NCTC 10437; (b) $M$. bovis NCTC 10772; (c) M. scrofulaceum NCTC 10803; (d) $M$. tuberculosis NCTC 7416; (e) $M$. tuberculosis TB1 (isoniazid resistant); (f) $M$. tuberculosis TB2 (isoniazid and streptomycin resistant); control (O) and NAP (O).

produced heavy growth in both conventional and radiometric media containing $P N B$.

NAP. M. tuberculosis NCTC 7416 and $M$. bovis NCTC 10772 were inhibited by NAP, whereas $M$. avium NCTC 10437 and $M$. scrofulaceum NCTC 10803 were resistant (fig. 3). In contrast to the results obtained in the PNB test, all isolates of $M$. kansasii grew rapidly in the presence of NAP (fig. 2).

TCH. M. tuberculosis NCTC 7416, three clinical isolates of $M$. tuberculosis and two of the three isoniazid-resistant strains of $M$. tuberculosis were resistant to $\mathrm{TCH}$ (fig. 4). In contrast, $M$. bovis NCTC 10772 and three $M$. bovis isolates from veterinary sources were all inhibited by TCH. $M$. avium NCTC 10437 and $M$. scrofulaceum NCTC 10803 were not inhibited by $\mathrm{TCH}$.

\section{Experience with clinical isolates}

During a 1-year period, 131 isolates of mycobacteria identified by conventional methods were tested by radiometric methods. The results obtained with the radiometric methods are given in the table. All isolates of the $M$. tuberculosis-bovis complex were inhibited by both PNB and NAP, whereas the 10 isolates of MOTT were uniformly resistant to both agents.
Of the strains identified as $M$. tuberculosis or $M$. bovis, 110 were resistant to $\mathrm{TCH}$ and were identified as $M$. tuberculosis; the remainder were inhibited by TCH and were identified as $M$. bovis, with the exception of one isolate of BCG. There was complete agreement between the radiometric and conventional methods of identification.

Two isolates of $M$. bovis and one isolate of $M$. tuberculosis were resistant to isoniazid. One other isolate of $M$. tuberculosis was resistant to streptomycin and isoniazid. Cross-resistance between isoniazid and $\mathrm{TCH}$ was not evident.

\section{Discussion}

Clinically significant isolates and reference strains of mycobacteria were successfully differentiated in this study by use of PNB and TCH as selective inhibitors in a radiometric technique. Since the observation by Tsukamura and Tsukamura (1964) that PNB was a useful differentiating agent for mycobacteria, several workers have used susceptibility to $500 \mu \mathrm{g} / \mathrm{ml}$ in LJ medium as a routine screening method. This investigation has demonstrated that a radiometric PNB test performs as well as the conventional PNB test and a commercially produced radiometric NAP test. 

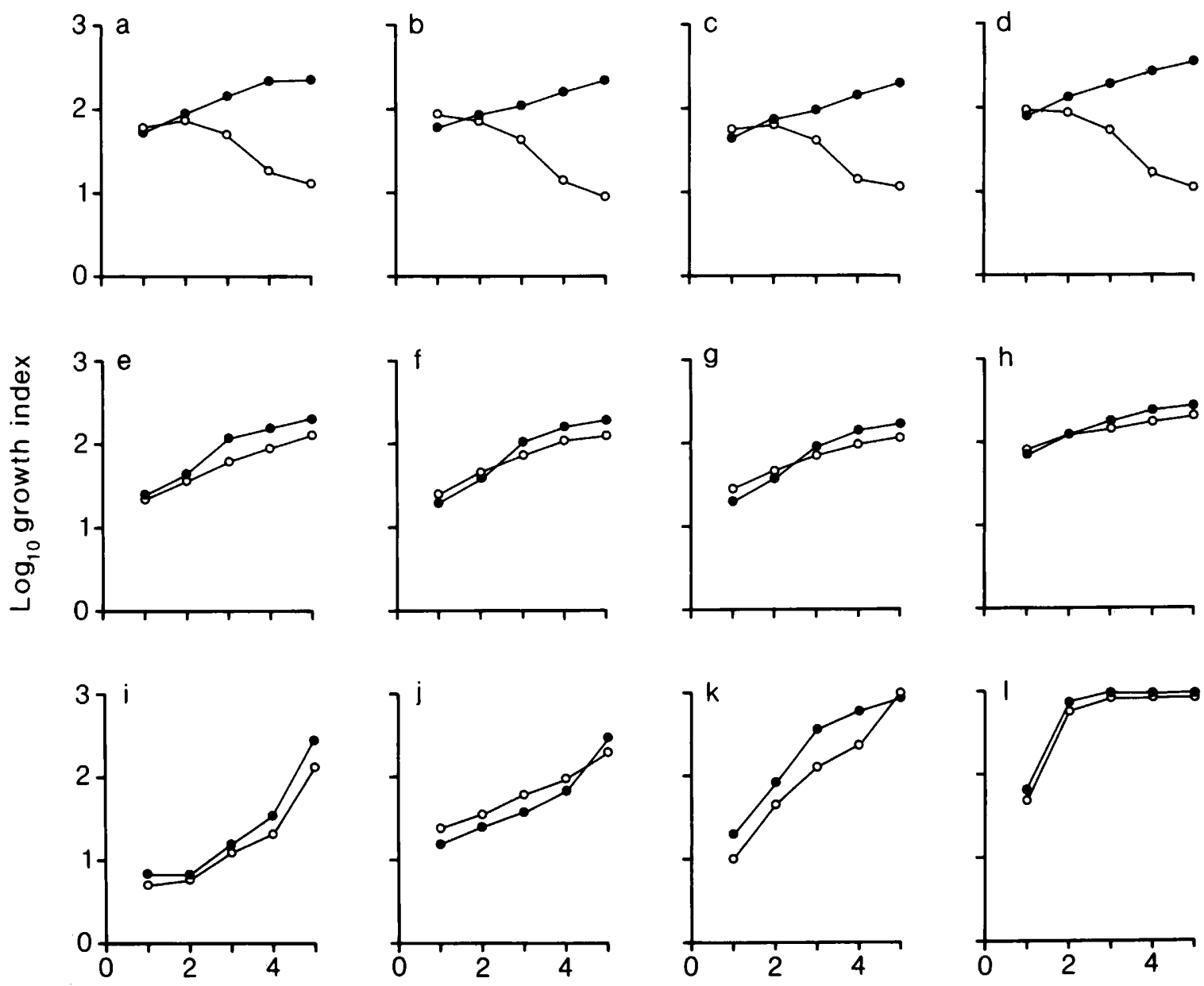

\section{Days}

Fig. 4. Susceptibility of mycobacteria to thiophene-2-carboxylic acid hydrazide (TCH): (a) $M$. bovis NCTC 10772; (b-d) $M$. bovis (recent veterinary isolates); (e) $M$. tuberculosis NCTC 7416; (f-h) $M$. tuberculosis (recent human isolates); (i) $M$. tuberculosis TB1 (isoniazid resistant); (j) M. tuberculosis TB2 (isoniazid and streptomycin resistant); (k) $M$. scrofulaceum NCTC 10803 ; (l) $M$. avium NCTC 10437; control (O) and TCH $1 \cdot 2 \mu \mathrm{g} / \mathrm{ml}(\mathrm{O})$.

Table. Identification of 131 isolates of Mycobacterium spp. by radiometric PNB, NAP and $\mathrm{TCH}$ inhibition tests

\begin{tabular}{|c|c|c|c|c|c|c|c|}
\hline \multirow[b]{3}{*}{ Species } & \multirow{3}{*}{$\begin{array}{c}\text { Number of } \\
\text { isolates }\end{array}$} & \multicolumn{6}{|c|}{ Results of inhibition tests with } \\
\hline & & \multicolumn{2}{|c|}{ PNB } & \multicolumn{2}{|c|}{ NAP } & \multicolumn{2}{|c|}{$\mathrm{TCH}$} \\
\hline & & $\mathbf{S}$ & $\mathbf{R}$ & $\mathrm{S}$ & $\mathbf{R}$ & $\mathbf{S}$ & $\mathbf{R}$ \\
\hline M. tuberculosis & 110 & 110 & 0 & 110 & 0 & 0 & 110 \\
\hline M. bovis & 10 & 10 & 0 & 10 & 0 & 10 & 0 \\
\hline $\mathrm{BCG}$ & 1 & 1 & 0 & 1 & 0 & 1 & 0 \\
\hline M. kansasii & 2 & 0 & 2 & 0 & 2 & 0 & 2 \\
\hline M. fortuitum & 2 & 0 & 2 & 0 & 2 & 0 & 2 \\
\hline M. avium/intracellulare & 3 & 0 & 3 & 0 & 3 & 0 & 3 \\
\hline M. gordonae & 2 & 0 & 2 & 0 & 2 & 0 & 2 \\
\hline$M$. xenopi & 1 & 0 & 1 & 0 & 1 & 0 & 1 \\
\hline
\end{tabular}

$\mathrm{S}=$ sensitive $; \mathrm{R}=$ resistant . 
A small proportion of isolates of $M$. kansasii and $M$. marinum will not grow in the presence of PNB (Tsukamura, 1970, 1981). In the present study, two strains of $M$. kansasii failed to grow in the radiometric $\mathrm{PNB}$ test and produced a scanty growth on conventional media containing PNB after incubation for 3 weeks. If sensitivity was interpreted as the complete absence of growth on LJ slopes, the conventional test would suggest that these strains were resistant to PNB. However, these strains would be considered susceptible if resistance was indicated only when growth of 20 or more colonies was evident, and the 19 colony $=$ susceptible $: 20$ colony $=$ resistant endpoint is commonly used in sensitivity tests on mycobacteria.

The PNB-susceptible $M$. kansasii strains were not inhibited by NAP; from the results presented above it would appear that NAP is less inhibitory than PNB for these strains. However, they grew slowly in the presence of NAP and high GI values were not attained. Difficulty in interpretation of NAP sensitivity results for strains of $M$. kansasii has been encountered by other workers, necessitating a longer incubation period of 5-7 days (Morgan et al., 1985). Although the NAP test would appear to be more sensitive than the PNB test for the identification of $M$. kansasii, it has been shown that not all strains of $M$. marinum, $M$. chelonei subsp. chelone $i$ and $M$. xenopi are resistant to NAP in the radiometric test (Laszlo and Siddiqi, 1984). Similar difficulties are encountered with the conventional NAP test if conditions are not carefully standardised, inoculum size and incubation temperature being particularly important (Laszlo and Eidus, 1978).

When 131 clinical isolates were examined, the radiometric $\mathrm{PNB}$ test was found to be as accurate as the NAP test for the differentiation of MOTT from the $M$. tuberculosis complex. PNB is less expensive than NAP, is more easily obtained and has for many years been a reliable selective agent

\section{REFERENCES}

Collins C H, Yates M D 1979 Hydroxylamine sensitivity and salt tolerance in screening and identifying mycobacteria. Tubercle 60: 91-94.

Collins, C H, Grange, J M 1987 Zoonotic implications of Mycobacterium bovis. In: Arbuthnott J P (ed) Animal health: the control of infection. Royal Irish Academy, Dublin, pp 1-7.

Eidus L, Diena B B, Greenberg L 1960 The use of $\rho$-nitro- $\alpha$ acetylamino- $\beta$-hydroxypropiophenone (NAP) in differentiation of mycobacteria. American Review of Respiratory Diseases 81 : 759-760.

Harrington R, Karlson A G 1966 Differentiation between in conventional media. The additional dilution of the inoculum for the NAP test (Morgan et al., 1985) is unnecessary for the PNB test, as the method is that used for sensitivity testing with other antimycobacterial agents.

The results obtained with the radiometric $\mathrm{TCH}$ test were similar to those obtained with the conventional LJ slope method. Preliminary work during the development of this test emphasised that it was important to use an actively metabolising inoculum with a GI of at least 999.

Although the number of isoniazid-resistant isolates of $M$. tuberculosis in this study was small, any cross-resistance with $\mathrm{TCH}$ did not interfere with the sensitivity of either the conventional or the radiometric test. In a comprehensive study of the relationship between resistance of $M$. tuberculosis to isoniazid and TCH, Yates et al. (1984) concluded that cross-resistance occurred infrequently. In conventional $\mathrm{TCH}$ tests, a $\mathrm{TCH}$ concentration of $5 \mu \mathrm{g} / \mathrm{ml}$ is used, whereas in the radiometric test sensitivity was greater with $\mathrm{TCH} 1.2 \mu \mathrm{g} / \mathrm{ml}$, allowing earlier detection of growth.

While the incidence of bovine tuberculosis is low in Ireland, the possibility of zoonotic transmission exists and will remain until eradication of tuberculosis from cattle is achieved. The epidemiological monitoring of bovine strains is essential for the mycobacteriology laboratory (Collins and Grange, 1987).

Marks (1976) noted that basic classification of mycobacteria is enhanced when drug susceptibility tests are combined with other screening methods. This study has demonstrated that radiometric methods of detection and susceptibility testing can be further supplemented with rapid, sensitive and accurate differential tests for presumptive identification.

We thank Professor J. Flynn for his support and encouragement and Kilian McDaid for drawing the figures.

Mycobacterium tuberculosis and Mycobacterium bovis by in vitro procedures. American Journal of Veterinary Research 27: 1193-1196.

Jenkins P A 1987 AIDS and the lung. British Medical Journal 295: 331 .

Laszlo A, Eidus L 1978 Test for differentiation of $M$. tuberculosis and $M$. bovis from other mycobacteria. Canadian Journal of Microbiology 24: 754-756.

Laszlo A, Siddiqi S H 1984 Evaluation of a rapid radiometric differentiation test for the Mycobacterium tuberculosis complex by selective inhibition with $\rho$-nitro- $\alpha$-acetylamino$\beta$-hydroxypropiophenone. Journal of Clinical Microbiology 19: 694-698.

Lévy-Frébault V, Pangon B, Buré A, Katlama C, Marche C, 
David H L 1987 Mycobacterium simiae and Mycobacterium avium- $M$. intracellulare mixed infection in acquired immune deficiency syndrome. Journal of Clinical Microbiology 25 : 154-157.

Males B M, West T E, Bartholomew W R 1987 Mycobacterium haemophilum infection in a patient with acquired immune deficiency syndrome. Journal of Clinical Microbiology 25 : 186-190.

Marks J 1976 A system for the examination of tubercle bacilli and other mycobacteria. Tubercle 57: 207-255.

Morgan M A, Doerr K A, Hempel H O, Goodman N L, Roberts, G D 1985 Evaluation of the $\rho$-nitro- $\alpha$-acetylamino- $\beta$ hydroxypropiophenone differential test for identification of Mycobacterium tuberculosis complex. Journal of Clinical Microbiology 21 : 634-635.

Tsukamura M 1965 Differentiation of mycobacteria by susceptibility to hydroxylamine and 8-azoguanine. Journal of Bacteriology 90 : 556-557.
Tsukamura M 1970 Screening for atypical mycobacteria. Tubercle 51 : 280-283.

Tsukamura M 1981 A review of the methods of identification and differentiation of mycobacteria. Reviews of Infectious Diseases 3: 841-861.

Tsukamura M $1984 \rho$-Nitrobenzoic acid medium(PNB medium) as an aid to differentiate tubercle bacilli from other mycobacteria. (A review). Kekkaku 59: 361-368 (in Japanese).

Tsukamura M, Tsukamura S 1964 Differentiation of Mycobacterium tuberculosis and Mycobacterium bovis by $\rho$-nitrobenzoic acid susceptibility. Tubercle 45 : 64-65.

Yates M D, Grange J M, Collins C H 1984 A study of the relationship between the resistance of Mycobacterium tuberculosis to isonicotinic acid hydrazide (isoniazid) and to thiophene-2-carboxylic acid hydrazide. Tubercle 65: 295299. 